\title{
Primary Cardiac B-Non-Hodgkin Lymphoma Disguised as a Pacemaker Endocarditis
}

\author{
Felix Fleißner ${ }^{1,2}$ Ulrich Molitoris ${ }^{1}$ Wiebke Rösler ${ }^{3}$ \\ 1 Department of Cardiac, Thoracic, Transplantation and Vascular \\ Surgery, Hannover Medical School, Hannover, Germany \\ 2 Institute for Molecular and Translational Therapeutic Strategies \\ (IMTTS), Hannover Medical School, Hannover, Germany \\ 3 Department of Hematology, Hemostasis, Oncology and Stem Cell \\ Transplantation, Hannover Medical School, Hannover, Germany \\ Thorac Cardiovasc Surg Rep 2018;7:e18-e20.
}

\author{
Christian Kühn ${ }^{1}$
}

\begin{abstract}
Keywords

- pacemaker endocarditis

- primary cardiac nonHodgkin lymphoma
\end{abstract}

Address for correspondence Felix Fleißner, MD, Department of Cardiac, Thoracic, Transplantation and Vascular Surgery, Hannover Medical School, Carl-Neuberg- Strasse 1, Hannover 30625, Germany (e-mail: fleissner.felix@mh-hannover.de).

\section{Introduction}

Despite improvements in design and implantation techniques of cardiac devices, infection remains a serious problem. Although the reported incidence of infection varies among studies with infection rates between $0.13^{1}$ and $19.9 \%^{2}$ reported, infection rates seem to have increased over the recent years. A study by Uslan et al showed an incidence of cardiac implant infections of 1.9 per 1,000 device-years ${ }^{3}$ however, infection rates seem to be rising faster than implantation rates. ${ }^{4}$ Like endocarditis in general, lead endocarditis is a malignant disease with a 1-year mortality of $12 \%$ for pocket infections and $25 \%$ for endovascular infections. ${ }^{5}$ Symptoms of a pacemaker endocarditis include erosion, sinus tracts, fevers, chills, and sepsis. These symptoms are rather specific but are not sensitive. As for positive blood cultures and valvular or lead vegetations, they are signs of more severe disease and with a worse prognosis. In this patient, all clinical parameters indicated a pacemaker endocarditis. As for the large vegetation found around the lead in the left and right atrium, an open surgical approach was used to extract the pacemaker wires.

\section{Case Description}

A 78-year-old patient received a DDD pacemaker a year prior to the recent admission due to an atrioventricular (AV) block II $^{\circ}$ (MOBITZ 2). Postoperative course was uneventful and he was discharged 4 days after primary admission. Echocardiography was not performed. He neither received a coronary angiogram despite known coronary artery disease with percutaneous transluminal coronary angioplasty (PTCA) and stent implantation of the left anterior descending and right coronary artery 6 years ago. He developed subfebrile temperatures, deterioration of the general condition, as well as peripheral edema and tachycardia within 3 weeks prior to the next admission. He was readmitted to a general hospital. The initial diagnosis was a suspected pacemaker endocarditis. Echocardiography seemed to confirm the diagnosis showing large vegetation in the right and left atrium, connected via an atrioseptal defect (-Video $\mathbf{1}$ ). The patient was placed on ampicillin and gentamycin. He was urgently referred to our clinic for surgical removal of the pacemaker wires and provisional pacemaker implantation. ${ }^{6}$ We performed a coronary received

February 2, 2018 accepted after revision March 21, 2018
DOI https://doi.org/ $10.1055 / \mathrm{s}-0038-1660807$. ISSN 2194-7635. (c) 2018 Georg Thieme Verlag KG
Stuttgart · New York

License terms

$\Theta(1) \Theta \Theta$ 
angiogram showing a mild progression of the known coronary artery disease, especially for the right coronary artery with high-grade stenosis before and after the stent. Transthoracic echocardiography confirmed the vegetation in the left and right atrium as well as pericardial effusion. Drawn blood cultures were negative; however, the patient showed an increased C-reactive protein of $122 \mathrm{mg} / \mathrm{dL}$ and leucocytes of $12.1 \mathrm{Tsd} / \mu \mathrm{L}$. Pacemaker readout showed a decline in ventricular and atrial sensing from 11.2 to $4 \mathrm{mV}$ and 4.0 to $2.0 \mathrm{mV}$, respectively. Impedance decreased accordingly for the atrium and the ventricle from $457 \Omega$ to $313 \Omega$ and $959 \Omega$ to $490 \Omega$, respectively. The patient was scheduled for urgent open pacemaker explanation via a median sternotomy, according to the size of the vegetation found in the atrium (-Video 1).

\section{Video 1}

Four-chamber view of the large tumor mass between the right and left atrium via an atrial septum defect. The tumor mass originates from the septum, infiltrating the atrial wall. Online content including video sequences viewable at: https://www.thiemeconnect.com/products/ejournals/html/doi/10-1055s-0038-1660807-tcsr-18-0298-v1.mp4.

After a median sternotomy, the patient was placed on cardiopulmonary bypass and the atrium was opened. A large mass was encountered (-Fig. 1A). Surprisingly, the vegetation

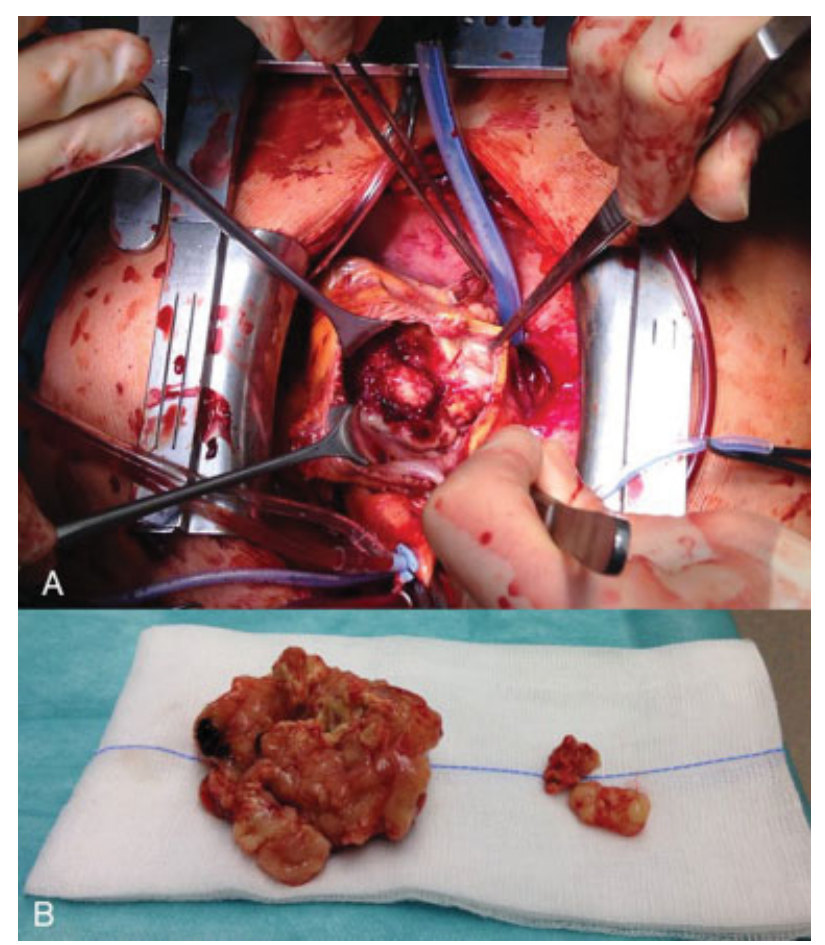

Fig. 1 (A) The large tumor mass after atriotomy; (B) the explanted tumor mass, which turned out to be a cell rich neoplasmatic malignant tumor. turned out to be of solid consistence and was highly tumor susceptive. Frozen section showed a cell rich neoplasmatic malignant tumor (-Fig. 1B). The patient received a tumor resection with a partial septum and atrial resection with an atrial septal defect closure, a reconstruction of the right atrium with a pericardial patch as well as an explanation of the DDD pacemaker wires and epicardial a left ventricular epicardial bipolar screwing electrode. The old pacemaker (which had still $\sim 8$ years of battery left) was connected to the epicardial pacemaker wires as VVI. Due to the extensive operation and the fact that the patient did not show any symptoms of coronary artery disease, no coronary artery bypass grafting was performed for the treatment of the right coronary artery stenosis. The patient was extubated timely on the intensive care unit. Postoperative course was uneventful and the patient was readmitted to the department of hematology and oncology the 12th postoperative day. Final pathology results of the explanted tumor showed a diffuse large cell B non-Hodgkin lymphoma stadium IE, IPI: 2. He further received three courses of R-Benda chemotherapy. Computed tomography after completion of the chemotherapy showed no indication for suspicious thoracic lymph nodes. In the following course, a decline in right ventricular (RV) sensing of the epicardial leads was detected and the patient received a RV and right atrial leads for DDD pacing. He was later discharged home where he recovered well from the operation. One year after the initial diagnosis, a reoccurrence of the B cell lymphoma occurred at the left dorsal thoracic wall and the hypopharynx. He again received chemotherapy. Today, 4 years after the initial diagnosis, he is tumor free in overall acceptable condition.

\section{Discussion}

In this patient, almost every clinical hint leads us to believe pacemaker endocarditis since the prevalence is significantly higher than a primary cardiac tumor. Especially the encounter of a primary cardiac lymphoma is a rare entity, especially in an immune-competent individual. Clinical presentation, fever, elevated C-reactive protein, and leucocytes as well as the initially found suspected vegetation in the right and left atrium lead to the suspicion of a pacemaker endocarditis. Nonetheless, as it turned out, the vegetation was a tumor rather than of infectious cause. This is to our knowledge the first description of a primary cardiac lymphoma disguised as a pacemaker endocarditis.

Retrospectively, as for primary cardiac tumors are known to cause arrhythmias, the initial development of the AV block could have been caused by the tumor developing a year prior to the recent event. We were surprised by the diagnosis of a tumor rather than a lead endocarditis. If we had performed a computed angiogram before the operation, we might have been able to identify the suspected vegetation as a tumor; however, the patient's symptoms as well as history lead us to believe in our initial diagnosis. Nonetheless, if we had been aware of the tumor, we probably would have chosen the same operative approach. This case is a clear exemption from the known saying: "if you hear hoof beats, think horses, not zebras." In fact, the primary non-Hodgkin lymphoma found in our patient was a quite rare breed of zebras. Altogether, our 
experience clearly showed that our preoperative diagnostics were inadequate.

Primary cardiac tumors are a rare disease with autopsy incidence ranging from 0.001 to $0.030 \%$. Most of the tumors (Three-quarters) are benign; nearly half the benign heart tumors being myxomas and the rest are lipomas, papillary fibroelastoma, and rhabdomyomas. ${ }^{7}$ Primary cardiac lymphomas are even less frequent with only a few cases reported over the years. ${ }^{8}$

A primary cardiac lymphoma is very rare with $\sim 60$ cases reported in the literature. ${ }^{9}$ To differentiate between a primary cardiac lymphoma and a cardiac involvement in generalized lymphoma (stage IV), complete clinical and radiological staging is needed: absence of lymphoma outside the pericardial sac after a complete autopsy examination and bulk of the neoplasm within the pericardium or cardiac symptoms from lymphomatous cardiac infiltration at the time of the initial diagnosis. ${ }^{10}$ The mean age at presentation for cardiac lymphoma is $\sim 38$ years with predominance in men. ${ }^{11}$ Patients with cardiac lymphomas present with various symptoms including cardiac tamponade, heart failure, exertional dyspnea, and atrial fibrillation as well as rightsided heart obstruction. ${ }^{12}$ Morphologically, the lymphomas show multiple, firm, whitish-yellow nodules. Histologically, they span the range of $B$ cell proliferations and include follicle center-cell lymphomas, immunoblastic lymphomas, diffuse large-cell lymphomas, and Burkitt lymphoma.

In conclusion, we hereby encountered an unexpected very rare entity of a primary cardiac lymphoma around the pacemaker leads.

\section{References}

1 Aljabri K, Garlitski A, Weinstock J, Madias C. Management of device infections. Card Electrophysiol Clin 2018;10(01):153-162
2 Bluhm G. Pacemaker infections. A clinical study with special reference to prophylactic use of some isoxazolyl penicillins. Acta Med Scand Suppl 1985;699:1-62

3 Uslan DZ, Sohail MR, St Sauver JL, et al. Permanent pacemaker and implantable cardioverter defibrillator infection: a population-based study. Arch Intern Med 2007;167(07): 669-675

4 Voigt A, Shalaby A, Saba S. Rising rates of cardiac rhythm management device infections in the United States: 1996 through 2003. J Am Coll Cardiol 2006;48(03):590-591

5 Jones SO IV, Eckart RE, Albert CM, Epstein LM. Large, singlecenter, single-operator experience with transvenous lead extraction: outcomes and changing indications. Heart Rhythm 2008;5 (04):520-525

6 Pichlmaier M, Knigina L, Kutschka I, et al. Complete removal as a routine treatment for any cardiovascular implantable electronic device-associated infection. J Thorac Cardiovasc Surg 2011;142 (06):1482-1490

7 Butany J, Nair V, Naseemuddin A, Nair GM, Catton C, Yau T. Cardiac tumours: diagnosis and management. Lancet Oncol 2005;6(04): 219-228Review

8 Burke A, Virmani R, for the Armed Forces Institute of Pathology (USA). Universities Associated for Research and Education in Pathology Neoplasms of the heart and great vessels, Armed Forces Institute of Pathology, Washington, DC (1996), p. 231

9 Anghel G, Zoli V, Petti N, et al. Primary cardiac lymphoma: report of two cases occurring in immunocompetent subjects. Leuk Lymphoma 2004;45(04):781-788

10 Ikeda H, Nakamura S, Nishimaki H, et al. Primary lymphoma of the heart: case report and literature review. Pathol Int 2004;54(03): 187-195

11 Sarjeant JM, Butany J, Cusimano RJ. Cancer of the heart: epidemiology and management of primary tumors and metastases. Am J Cardiovasc Drugs 2003;3(06):407-421

12 Burke A, Virmani R. Tumours and tumour-like conditions of the heart. In: Silver MD, Gotleib AG, Schoen FJ, eds. Cardiovascular Pathology. New York: Churchill Livingstone; 2001: 583-605 